\title{
A Document based Framework for Smart Object Systems
}

\author{
Fahim Kawsar, Tatsuo Nakajima \\ Waseda University, Japan \\ $\{$ fahim,tatsuo\}@dcl.info.waseda.ac.jp
}

\author{
Jong Hyuk Park \\ Kyungnam University, Korea \\ parkjonghyuk1@hotmail.com
}

\author{
Young-Sik Jeong \\ Wonkwang University, Korea \\ ysjeong@wonkwang.ac.kr
}

\begin{abstract}
We present an architectural framework that provides the foundation for building smart object systems and uses a document centric approach utilizing a profile based artefact framework and a task based application framework. Our artefact framework represents an instrumented physical smart objects as a collection of service profiles and expresses these services in generic documents. Applications for smart objects are expressed as a collection of functional tasks (independent of the implementation) in a corresponding document. A runtime component provides the foundation for mapping these tasks to the corresponding service provider smart objects. This mapping is spontaneous and thus enables gradual addition of services. Primary advantages of our approach are twofold-firstly, it allows developers to write applications in a generic way regardless of the constraints of the target environment. Secondly, it allows extension of functionalities of smart objects and applications very easily. We describe an implemented prototype of FedNet, and show examples of its use in a real life scenario to illustrate its feasibility.
\end{abstract}

\section{Introduction}

One of the consequences of pervasive technologies (e.g., miniaturization of the computer technologies and proliferation of wireless internet, short-range radio connectivity, etc.) is the integration of processors and tiny sensors into everyday objects. This revolutionized our perception of computing. We are in an era, where we communicate directly with our belongings, e.g., watches, umbrella, clothes, furniture or shoes and they can also intercommunicate. These everyday objects are designed to provide supplementary services beyond the primary purpose, an initiative that has been denoted as Smart Object ${ }^{1}$ computing. It

\footnotetext{
${ }^{1}$ In this paper, smart objects and augmented artefacts carry similar meaning and will be used interchangeably.
}

has drawn significant attention from the research community, primarily because of its promising potential in various industries e.g., supply chain management, medicine, environment monitoring, entertainment, smart spaces, etc.

In this paper, we look at the system issues for these smart objects. In particular we discuss two issues 1) a suitable artefact framework for representing smart objects and 2) a middleware that enables the rapid development of smart object systems. Primarily, we will look at the appropriate abstraction that we can use in smart object systems to provide a framework that enable rapid development of portable and extensible smart objects systems. The basic idea of our framework is to use documents to externalize an application's requirements in a generic way without considering smart object management issues. Similarly, smart objects' services are externalized by structured documents. A runtime infrastructure provides a semantic association between the applications and smart objects using structural type matching of these documents. Because of such loose coupling, applications and smart objects can be built and extended orthogonally.

\section{Motivation and Design Issues}

Smart object systems usually involve sensor instrumented everyday objects, mobile devices, displays, etc. to provide some user-centric proactive services. Our framework provides support for building this class of application in a simplistic way by utilizing a document centric approach. Our design has been influenced by two successful approaches existing currently. First one is the internet which is an excellent example of document based system. The internet is a collection of millions of anonymously authored digital documents that are encoded in a pre defined semantics that enable heterogenous platforms to exchange these documents. The fundamental issue here is the pre negotiation of the semantics. The most widely used protocol for internet, i.e., HTTP is basically acts as the en- 


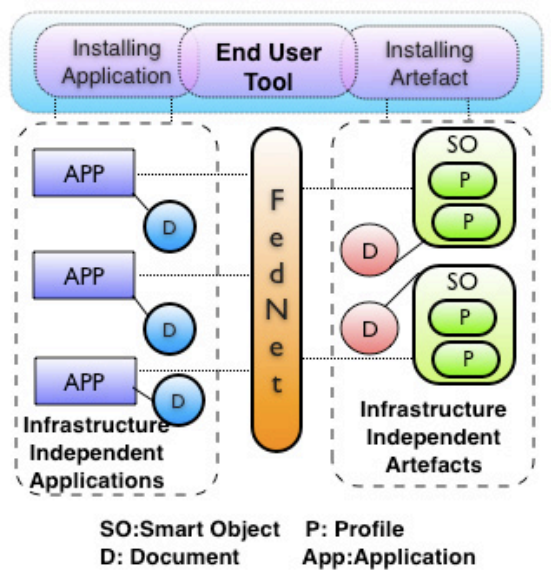

(a)

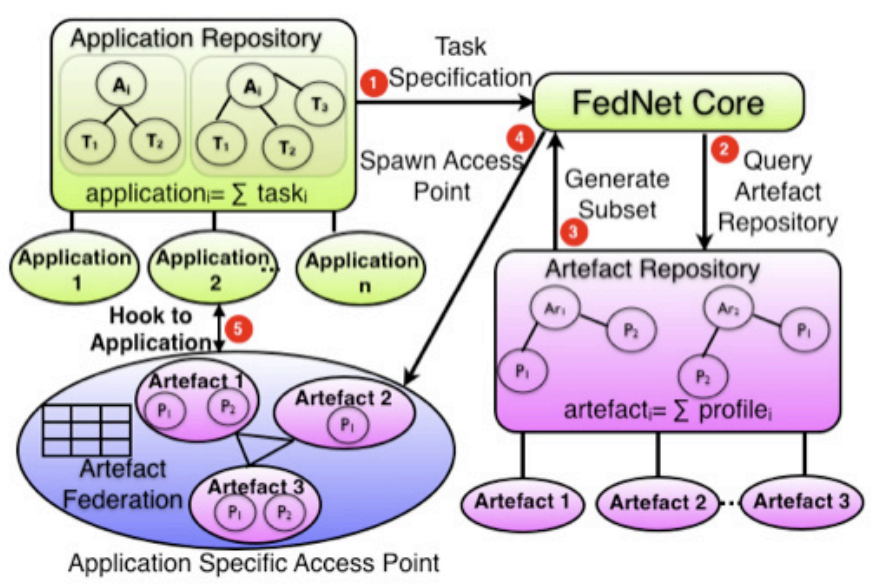

(b)

Figure 1. (a) Basic workflow of our approach (b) The internal architecture of FedNet

velope for this documents and provides the negotiation semantics to both the sender and recipient (i.e., servers and client browsers and vice versa) through it headers for a flawless communication. Henceforth, structured document is the primary resource and HTTP (headers) acts as the connecting glue in the internet infrastructure. In our approach, we consider applications are the consumers and smart objects are the resources. Thus if both are expressed and amended with pre negotiated semantics using documents like HTTP headers, we can easily provide a runtime association. The second influencing approach is the commonly used shell scripting to connect arbitrary programs using the UNIX pipe facility [6] where file handles (i.e., stdin, stdout, stderr) are used to differentiate and route data. From an abstract view point we can observe that this capability of semantic mapping by pipe facility is basically the negotiation of input/output structure. Thus, a structured document with pre negotiated semantics can perform the similar piping between application and smart objects. Henceforth, documents can glue an application with smart objects given the fact that they have pre negotiation through some abstract notions. Thus our primary challenge in document based approach is to provide appropriate abstraction underneath the documents that can be utilized to build smart object systems. With these viewpoints we have designed our framework adopting following design guidelines:

1. Providing appropriate wrapper framework and abstraction with structured documents to build smart objects without concerning the target application requirement.

2. Providing appropriate abstraction with structured documents for application developers using which they can externalize application's requirements and utilize smart objects without concerning interfaces and the management of smart objects.

3. Utilizing a runtime intermediator that handles smart object management (Bootstrapping, Discovery, Utilization) and provides mapping between application and smart object services based on structural type matching thus separating the concerns of the application and the middleware.

4. Providing service extension support for both the application and the smart object using primary abstractions.

These design principles enable developers to write applications and to build smart objects in a generic way regardless of the constraints of the target environment. The runtime infrastructure provides the pairwise mapping using structural typing thus externalizing smart object management and addressing heterogeneity issues away from the applications allowing developers to focus on the application functionalities only. This results in simple and rapid development of smart object systems.

\section{System Architecture}

Our middleware consists of an Artefact Framework, an Application Development Model and a Runtime Intermediary Infrastructure called FedNet. The basic workflow of our middleware is shown in Figure 1 (a). Artefact framework represents a smart object by encapsulating its augmented functionalities (e.g., proactivity of the table lamp) in one or multiple service profiles atop a runtime and allows additions of profiles incrementally. Applications in our approach are represented as a collection of implementation independent functional tasks. These tasks are atomic actions that represent the smart objects' services. An infrastructure component FedNet, manages these applications and artefacts and 


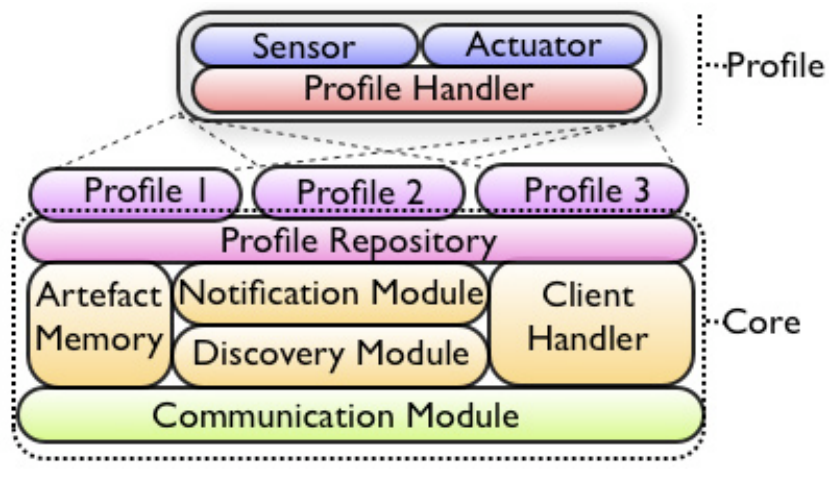

Figure 2. Architecture of Artefact Framework

maps the task specifications of the applications to the underlying artefacts' services by matching respective documents (that express the applications and the artefacts). Primarily these two abstractions Profile and Task are used in our system and realized by corresponding documents. Additionally end user tool can be built atop our middleware independently to deploy, configure and manage the applications and the artefacts.

\subsection{Artefact Framework}

Artefact framework provides a layered architecture as shown in Figure 2 where basic smart object functionalities are combined in a generic core component that act as the runtime. Additional augmented features can be added as plug-ins into the core. Each augmented feature is called a Profile in our approach. These profiles are artefact independent and represent a generic service implementing service specific protocols., For example: sensing room temperature could be one profile, and multiple artefacts (e.g., a window, an air-conditioner, etc.) can be augmented with a thermometer for supporting this profile. A profile handles the service specific heterogeneity by hiding the implementation detail and can be plugged into the runtime core of our artefact framework, allowing any profile to be a part of a suitable artefacts. The runtime core of a smart object hosts an array of its profiles and provides the basic communication facilities (e.g., service advertisement, pushing/pulling profile services to/from respective applications). A smart object and its service profiles are externalized using structured documents expressed in XML as shown in Figure 3. This document specifies the profile detail, i.e., input/output data type, methods, parameters, etc.

\subsection{Task Centric Application Model}

Any application is composed of several functional tasks, i.e., atomic actions. In smart object systems, these atomic actions may be: "turn the air-conditioner on", "sense the proximity of an object" etc. In our application model, an

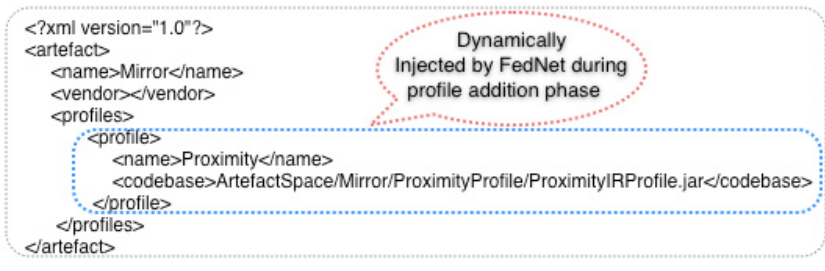

\section{Figure 3. Artefact Description File for a Mirror with Proximity Profile}

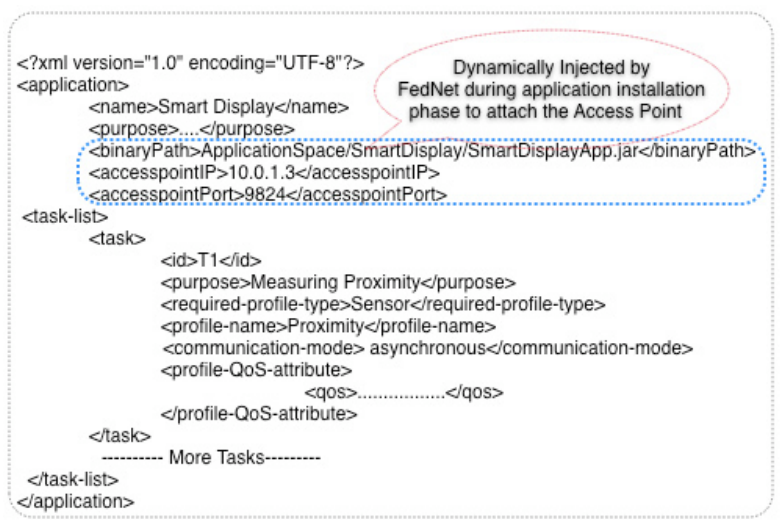

Figure 4. Task Description File (partly) for a smart display application

applications atomic actions are externalized as Tasks in a corresponding Task Description File expressed in XML as shown in Figure 4. This document explicitly specifies the service requirements of this application and each task in the document specifies the respective profiles of the smart objects it needs to accomplish its goal. The next feature of our application model is the interface definition. We consider defining strict interfaces for applications limits the portability and adoption of applications. Since any application only needs to manipulate data to interact with underlying smart objects, a compatible and consistent message is enough to enable applications interaction with smart objects. Consequently, we have addressed this concern by allowing FedNet, the intermediary component of our middleware to act as the gateway of smart objects services and accessing those services from application in a RESTful manner using simple HTTP get and post with XML messages. Thus applications do not need to adhere any middleware specific interfaces to interact with the smart objects yet can leverage their services via FedNet.

\subsection{FedNet Runtime Infrastructure}

In our approach both the applications and artefacts are infrastructure independent and expressed in high level de- 
scriptive documents (i.e., task and profile specifications). FedNet provides the runtime association among them by utilizing only the documents of these applications and artefacts. It can contact the the artefact core using the semantics described in the artefact documents for mapping application tasks, similarly application can contact FedNet in a RESTful manner. FedNet itself is packaged in a generic binary and composed of four components, Figure 1(b) shows the interaction among four components.

1. Application Repository hosts all the applications that run on FedNet. During an application's deployment, the binary executable and the Task Description File (TDF) are submitted to this repository. FedNet Core generates the an access point for the application and updates the respective TDF by dynamically injecting the identity of the corresponding access point as shown in Figure 4.

2. Artefact Repository manages all the artefacts running in FedNet environment. During artefacts' deployment, the executable binary implementing the artefact framework and the Artefact Description File (ADF) are submitted to this repository. When a profile is added to an artefact, the profile information is dynamically injected into ADF as shown in Figure 3 and the respective profile is attached to the artefact.

3. FedNet Core provides the foundation for the runtime federation. When an application is deployed the task specification is extracted from the application repository by the FedNet Core. It analyzes the task list by querying the artefact repository and generates an appropriate template of the federation and attaches it into a generic access point component for that application. When an application is launched, the access point is instantiated and the respective template is filled by the actual artefact available in the environment right at that moment thus forming a spontaneous federation.

4. Access Point represents the physical environment needed by an application. Since each application's artefact requirement is different and each application might not be running all the time, FedNet assigns a unique access point for each application; meaning multiple federations of artefacts can co-exist in the environment. Simultaneously, each artefact can participate in multiple federations. When an application is launched, the access point sends the federated artefacts data semantics to the application. This allows an application to know the semantics of movable data in advance. From then on, the application delegates all its requests to the access point which in turn forwards them to the specific artefact. The artefacts' responses to these requests by providing their profile outputs either by pushing the environment state (actuation) or

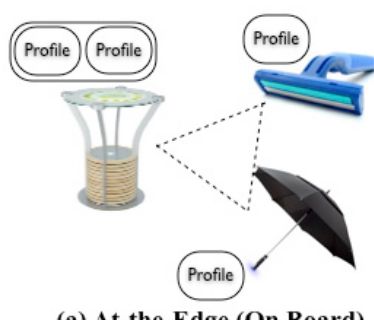

(a) At-the-Edge (On Board)

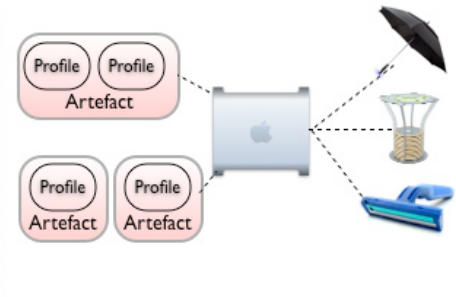

(b) At-the-Infrastructure (Off Board)

\section{Figure 5. Location Modalities of Artefact Framework}

pulling the environment states (sensing) back to the access point that are fed to the application.

\subsection{Distributed Management}

In the earlier part of this section we have provided the explanation of the functional roles of the primary components of our infrastructure. From physical implementation point of view all these components could be distributed, i.e., instrumented artefacts can run in their own nodes, applications can run on the artefact nodes, or in a separate node integrating multiple artefact nodes, and FedNet can run in its own node to manage all other nodes. The artefact framework essentially is the digital identity of an artefact. So an obvious issue is the location of this digital part. We have two choices as shown in Figure 5: a) At-the-Edge (On-Board) b) At-the-Infrastructure (Off-Board). At-the-Edge means the artefact itself has a processing unit that hosts its digital representation where as the At-the-Infrastructure means a proxy, running in a separate location represents the artefacts and communicates with the artefact to retrieve sensor data or to actuate artefact's function using some communication protocol, e.g., Bluetooth, IEEE 802.11x, etc. Both choices have pros and cons. While at-the-edge approach provides pre-configurable and self sustainable artefacts, it is prone to limited capability. On the other hand, although at-theinfrastructure approach requires manual configuration and maintenance, the primary advantage is the rapid prototyping support. In our current implementation we have adopted Atthe-Infrastructure approach and each artefacts digital representation, i.e., artefact framework's binary core and profile plug-ins are deployed in a node that communicates with the physical artefact thr-ough some communication channel to retrieve the actual profile service via the hardware attached into the artefact. The same is true for the applications, i.e., the applications running on a single artefact can reside in the same node that represents the artefact and the application that integrates multiple artefact can reside on the any of those artefacts node. It is the FedNet components that organize these nodes in a distributed manner and manages the 
spontaneous federation. The FedNet components (i.e., Application Repository, Artefact Repository and FedNet Core) can reside in one or multiple nodes and manage the underlying artefacts and applications.

\section{An Application Scenario}

We have built several smart object systems using our middleware. In this section we are presenting one of those systems.

We constructed a smart mirror by augmenting a regular laptop with acrylic magic mirror. Initially this mirror has a display profile. We wrote an application for this mirror where the application can show some personalized information (e.g. weather, stock quote, movie listing etc.) into the mirror display. However, this application can proactively show information only when someone is in-fornt of the mirror. But for such proactivity it requires a proximity profile. To enable this application feature, later we have added a proximity profile to this mirror by attaching an Infra-red sensor. This improved the applications interactivity. Afterwards we built a completely separate application for the mirror where user's dental hygiene is reported in a persuasive way utilizing the metaphor of a clean and dirty aquarium. We replaced the previous application running on the mirror with the new one. This new application requires a smart toothbrush that can detect its state-of-use. We constructed the smart toothbrush by attaching a wireless accelerometer sensor and deployed it in our environment with corresponding profile. Thus the mirror shows an aquarium reflecting users brushing practice whenever the user brushed his teeth in front of the mirror. All the smart objects and applications were deployed and configured using our end user deployment tool running atop FedNet. We have also performed an informal user study for evaluating the usability of our approach form end users point of view that we have reported at other forum [5].

Both the applications were built independently and deployed with corresponding documents expressing the tasks, similarly the two smart objects e.g., the toothbrush and the mirror were built independently and deployed with corresponding documents. FedNet provided the runtime association among them thus freeing application from smart object management. Furthermore, this scenario highlighted the service extension feature of our middleware. We have added new profiles to an existing smart mirror allowing an existing application to leverage new functionalities. Importantly, the application did not have to take into account the heterogeneity issues introduced by the addition of an Infrared sensor as it was handled by the proximity profile implementation.

\section{Discussion}

There are primarily two abstractions that we have utilized in our middleware. From the smart objects' perspec- tive it is the notion of profile that handles the service implementation detail and protocol issues. Since profiles are independently built following a plugin architecture, a smart object service can be extended anytime by adding new sensors or actuators and attaching corresponding profile into the smart objects core. Also, if a specific service needs to be updated only the corresponding profile need to be replaced, not the entire smart object or the applications utilizing them. Furthermore, a profile may provide services in various granularities thus supporting multiple applications requiring services at different scale (i.e., some applications may ignore some service features). The second abstraction is from applications' perspective, i.e., tasks that simply externalize an applications requirements, so any application can be expressed with this abstraction. Not necessarily all tasks of an application can be supported by an existing environments, however with the incremental addition of new smart objects in the environment or porting application to another environment with richer smart objects might enable the full functional features of an application. In addition an applications functionalities can be updated independently (application binary and the document) without concerning the impact of such update in the middleware or smart objects. In our approach, such flexibilities are provided elegantly by only expressing applications' task specifications in documents and ignoring smart object management issues at the application level. FedNet provides the appropriate mapping of these documents with smart objects documents expressing their services. This disassociation of applications from the smart objects they reference is identical to the Model-View-Controller (MVC) architecture from Smalltalk. In the MVC architecture, data (the model) is separated from the presentation of the data (the view) and events that manipulate the data (the controller). Similarly, documents in our middleware act as the glue that associates smart objects services to applications that manipulate the services. Such separation of concern (i.e., both the applications are artefacts are independent of FedNet and come as ready-to-run binary), and data centric approach also enable us to provide additional services orthogonally in our system. For example, we have implemented several end user tools atop FedNet that enable end users to deploy, configure and manage the applications and smart objects running in the FedNet environment.

\section{Related Work}

To date several methods have been proposed to address system support for ubicomp applications. One approach is interface and protocol standardization as attempted by $\mathrm{Jini}^{2}$ and $\mathrm{UPnP}^{3}$ respectively. Jini describes devices using interface description and language APIs allowing applications to

\footnotetext{
${ }^{2}$ Jini - http://www.sun.com/software/jini

${ }^{3}$ Universal Plug and Play - http://www.upnp.org
} 
utilize those interfaces where as UPnP attempts to standardize protocols to allow devices to intercommunicate seamlessly. These infrastructures provides well defined interfaces for application developers, however it is hard to build application integrating appliances that do not follows their specific protocols. Furthermore, these systems provide little support for extending applications or appliance services. For example, it is hard to add features in an existing artefact and using that feature immediately in the application with these infrastructures. Patch Panel [1] is a programming tool that provides a generic set of mechanisms for translating incoming events to outgoing events using EventHeap [4] communication platform. It allows new applications to leverage the services of existing components. Our overall approach is close to Patch Panel as we seek to support incremental integration. However, we exploit a distributed state model with an artefact framework that enable incremental addition of features to both artefacts and applications. In SpeakEasy [3] mobile codes (typed data streams and services) are exchanged among heterogeneous devices to create an interoperable environment. SpeakEasy does not consider the incremental extension of artefact services or end user deployment as its primary focus is on service composition. InterPlay [7] is a home $\mathrm{A} / \mathrm{V}$ device composition middleware and uses pseudo sentences to capture user intent, which is converted into a higher level description of user tasks. These tasks are mapped to underlying devices that are expressed using device description. Although our approach is very close to InterPlay as we employ similar mapping of tasks to device services, our challenge is to provide generic abstractions and to support incremental extension and deployment of both artefacts and applications. Our artefact framework is a major leap from InterPlay which signifies our contribution. A range of middlewares for pervasive systems $[2,8,9]$ specify their application development processes strictly. These middlewares usually provide end-to-end support for the application developer, i.e., instrumented artefacts are encapsulated into wrappers and an array of APIs is provided to the applications to manipulate them. The problem of this approach is that the applications and the instrumented artefacts become virtually incompatible in other environments. We have adopted a document centric approach allowing development of infrastructure independent applications and artefacts and the runtime association between them is provided by FedNet.

\section{Conclusion}

In this paper we have presented a document based approach to build smart object systems. Applications' requirements and smart objects' services are externalized using structured documents utilizing Task and Profile abstractions respectively. A runtime framework FedNet provides the dynamic association by structural type matching. The contributions of our approach are two-fold: firstly, it allows developers to write applications in a generic way regardless of the constraints of the target environment utilizing the abstractions that are realized through documents Secondly, it allows extension of functionalities of smart objects and applications very easily. We have described an implemented prototype of our approach with an application scenario that highlight the power and flexibility of our framework. We consider our approach is very useful for the ubiquitous computing domain, particularly one that involves smart objects.

\section{References}

[1] R. Ballagas, A. Szybalski, and A. Fox. Patch panel: Enabling control-flow interoperability in ubicomp environments. In PerCom 2004, 2004.

[2] A. K. Dey, G. Abowd, and D. Salber. A conceptual framework and a toolkit for supporting the rapid prototyping of context-aware applications. Human Computer Interaction, 16(2-4):97-166, 2001.

[3] W. K. Edwards, M. Newman, J. Sedivy, T. Smith, and S. Izadi. Challenge: recombinant computing and the speakeasy approach. In th ACM MobiCom, 2002.

[4] B. Johanson, A. Fox, and T. Winograd. The interactive workspaces project: experiences with ubiquitous computing rooms. IEEE Pervasive Computing, 1-2, 2002.

[5] F. Kawsar, K. Fujinami, and T. Nakajima. Deploy spontaneously: Supporting end-users in building and enhancing a smart home. In The Tenth International Conference on Ubiquitous Computing (Ubicomp 2008), 2008.

[6] S. J. Leffler, M. K. McKusick, M. J. Karels, and J. S. Quarterman. The Design and Implementation of the 4.3 BSD UNIX Operating System. Addison-Wesley, Reading, MA, 1989.

[7] A. Messer, A. Kunjithapatham, M. Sheshagiri, H. Song, P. Kumar, P. Nguyen, and K. H. Yi. Interplay: A middleware for seamless device integration and task orchestration in a networked home. In IEEE PerCom 2006.

[8] M. Roman, C. K. Hess, R. Cerqueira, A. Ranganathan, R. H. Campbell, and K. Nahrstedt. Gaia: A middleware infrastructure to enable active spaces. IEEE Pervasive Computing, pages 74-83, 2002.

[9] J. P. Sousa and D. Garlan. Aura: an architectural framework for user mobility in ubiquitous computing environments. In 3rd Working IEEE/IFIP Conference on Software Architecture, 2002. 